CMEARTICLE

\title{
Clinics in diagnostic imaging (194)
}

Poh Yong $\underline{T a n}^{1}$, MBBS, MRCP, Wei Ping Tham ${ }^{2}$, MBChB, FRCR, Yan Rong Yong ${ }^{3}$, MBBS, FRCR

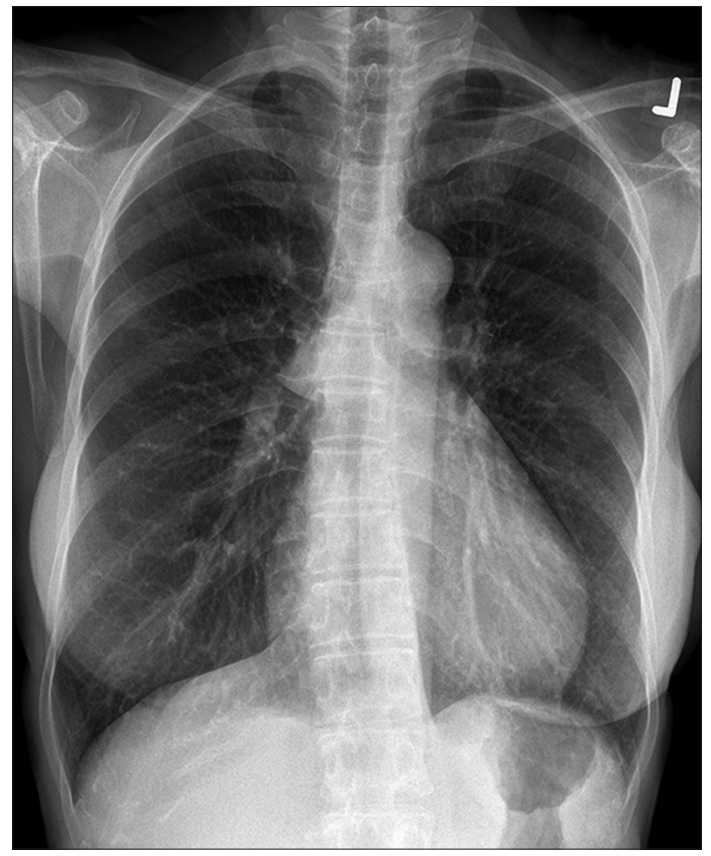

Fig. 1 Posteroanterior chest radiograph.
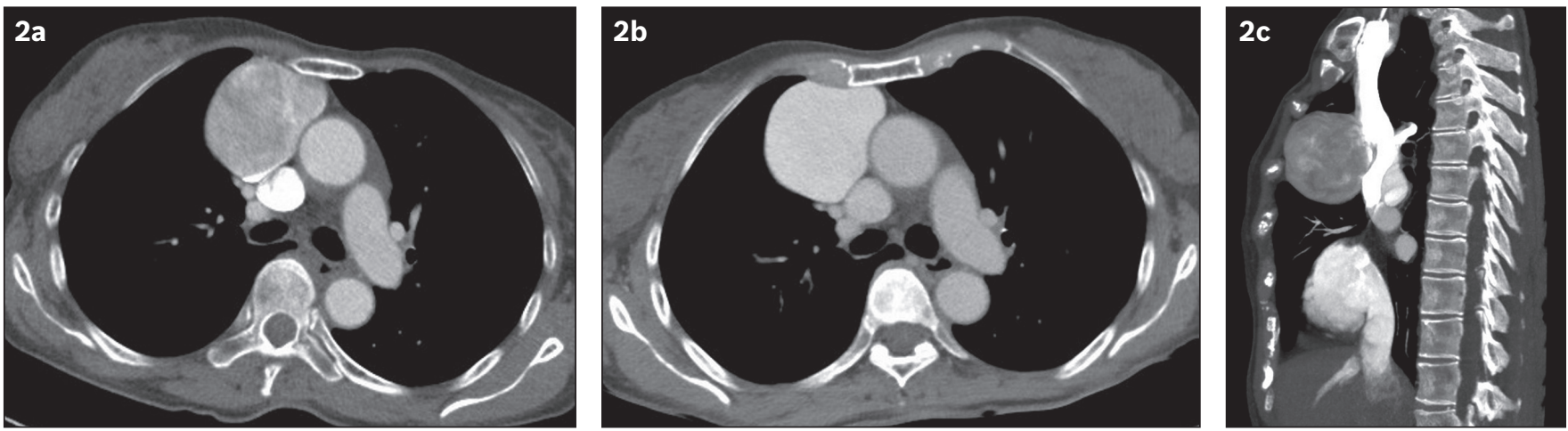

Fig. 2 (a) Arterial and (b) venous phase CT images of the chest. (c) Sagittal maximum intensity projection image of a CT aortogram.

\section{CASE PRESENTATION}

A 68-year-old woman presented with weight loss of $7 \mathrm{~kg}$ over two years. Colonoscopy and gastroscopy findings were normal. Clinical examination revealed no specific abnormality and the patient was otherwise asymptomatic.
Chest radiography (Fig. 1) was performed and showed an abnormality. Contrast-enhanced computed tomography (CT) of the chest (Fig. 2) was performed for further evaluation. What do these images show? What is the diagnosis?

${ }^{1}$ Department of Internal Medicine, ${ }^{2}$ Department of Diagnostic Radiology, Singapore General Hospital, ${ }^{3}$ Department of Diagnostic Radiology, Changi General Hospital, Singapore Correspondence: Dr Poh Yong Tan, Consultant, Department of Internal Medicine, Singapore General Hospital, Level 4, Academia, 20 College Road, Singapore 169856. tan.poh.yong@singhealth.com.sg 


\section{IMAGE INTERPRETATION}

The chest radiograph (Fig. 1) shows a subtle soft tissue opacity projected over the carina and right hilum, suggestive of a mediastinal mass. The hilum overlay sign is present, indicating that the hilum is visible through the mass and suggesting that the mass is not at the hilum. The vertebrae are also normal, favouring an anterior mediastinal location. CT image of the chest (Fig. 2) shows a large mass in the anterior mediastinum abutting the superior vena cava (SVC). The mass has a smooth outline with no invasion of surrounding structures. In the arterial phase, it demonstrates a swirling, heterogeneous enhancement pattern with contrast pooling at the dependent region. In the venous phase, the enhancement becomes homogeneous.

\section{DIAGNOSIS}

SVC aneurysm.

\section{CLINICAL COURSE}

The patient was asymptomatic and had no complications such as thrombosis, rupture or pulmonary embolism. As she was not keen on intervention, a decision was made for conservative management and follow-up CT of the chest to monitor for interval increase in the size of the mass and complications. Follow-up CT done seven months later showed the mass to be stable in size and appearance, with no complications.

\section{DISCUSSION}

SVC aneurysms are extremely rare, with only 43 cases reported to date. ${ }^{(1)}$ They are usually asymptomatic and discovered incidentally, as in our case. It has been postulated that congenital weakness in the SVC wall or absence of the longitudinal muscle layer in the tunica adventitia may predispose a patient to development of this entity. Post-trauma or postoperative causes (e.g. after a Glenn shunt or Fontan procedure) have also been reported. SVC aneurysms may be fusiform or saccular in shape, with saccular aneurysms being less common. Only 15 cases of saccular aneurysms have been reported to date. ${ }^{(1)}$

These lesions can be complicated by rupture, ${ }^{(2)}$ compression of surrounding structures, thrombus formation and subsequent pulmonary embolism, (3) especially in saccular aneurysms. There are no established guidelines on the management of SVC aneurysms. It has been suggested that asymptomatic aneurysms with diameters $<40 \mathrm{~mm}$ can be managed conservatively with periodic scans and anticoagulation. ${ }^{(4)}$ This is especially so for fusiform aneurysms that have lower complication rates. ${ }^{(5)}$ Thrombolytic therapy with recombinant tissue plasminogen activator was deemed to be harmful in one case, in which mobilisation of the thrombus in the SVC aneurysm resulted in recurrent pulmonary embolism. ${ }^{(3)}$ Surgical resection, usually through a median sternotomy approach, has been advocated for saccular aneurysms due to risks of complications, (1) and for aneurysms that are larger than $40 \mathrm{~mm}$, growing or symptomatic, or those that contain thrombi. ${ }^{(4)}$
Cardiopulmonary bypass should be used when operating on saccular or complex aneurysms, such as those with venous obstruction, calcification or thrombus formation, to prevent pulmonary embolism. ${ }^{(4,6)}$ Endovascular approaches can be considered for patients with high surgical risk. In one case report, a stent was deployed into the SVC and stent meshes were then used for coil occlusion of the neck of the aneurysm sac. ${ }^{(7)}$ Another case was described in which balloon-protected transcatheter thrombin injection was used in the management of a large saccular SVC aneurysm. ${ }^{(8)}$

SVC aneurysms are difficult to differentiate from other mediastinal masses such as the classical four T's of anterior mediastinal masses, namely thymoma, teratoma, thyroid nodule and terrible lymphoma. Contrast-enhanced CT can be used to differentiate the various lesions. On chest radiographs, SVC aneurysms usually appear as anterior mediastinal masses that are commonly projected over the right hilum but have the hilum overlay sign, suggesting an anterior relation to the hilum. The hilum overlay sign refers to the hilum being visible through the mass on radiographs; this implies that the mass is not at the hilum itself, as it would obliterate the silhouette of the hilum. On CT, the enhancement pattern of the SVC aneurysm is similar to that of the SVC, and contrast layering is seen in the dependent portion of the aneurysm in the arterial phase before filling the whole aneurysm in the venous phase, likely due to slow flow. Bubbles within the mass, due to gas inadvertently introduced into the bloodstream during injection of the contrast medium, are also a clue to the diagnosis of a vascular lesion. (1) The communicating channel with the SVC is not always seen but can appear as a stalk-like channel. ${ }^{(9)}$

In contrast, a mediastinal tumour such as a thymoma typically manifests as a homogeneously enhancing anterior mediastinal mass with smooth or lobulated contours, located anywhere from the thoracic inlet to the cardiophrenic angle. Heterogeneity due to calcification, necrosis, cystic change (Fig. 3) or haemorrhage can occur. In more aggressive subtypes of thymoma, invasion of surrounding structures or metastases is seen. ${ }^{(10)}$

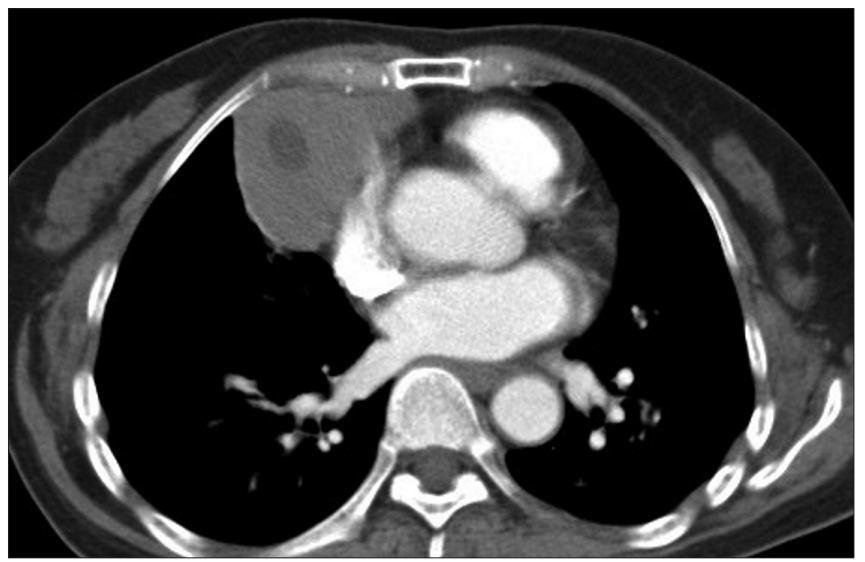

Fig. 3 A mediastinal mass found in a 73-year-old woman on routine chest radiography. Axial contrast-enhanced CT image shows a well-defined mass at the anterior mediastinum with generally homogeneous soft tissue density and a central cystic focus. The enhancement along the lateral surface of the mass is a vessel. Thymectomy was performed and a diagnosis of thymoma was made histologically. 


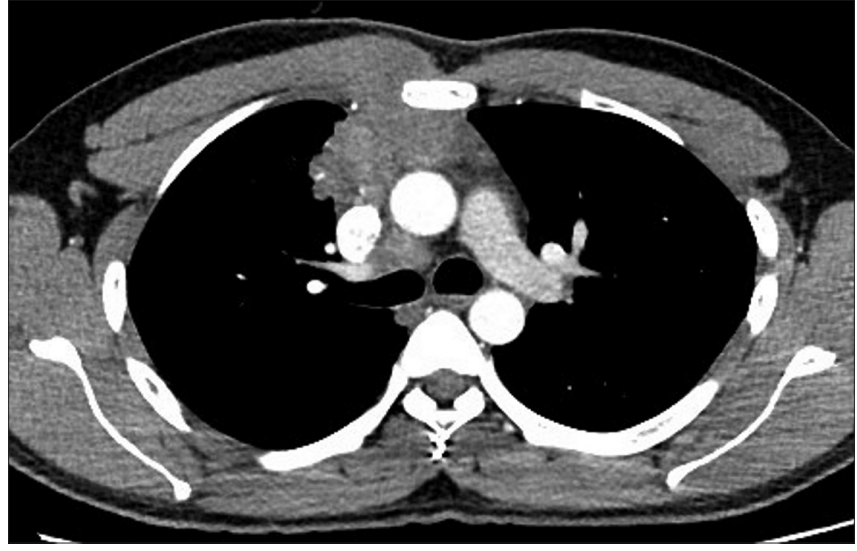

Fig. $4 \mathrm{~A}$ palpable mass at the right parasternal region in a 31-year-old man. Axial contrast-enhanced CT image shows an irregularly outlined soft tissue density mass, which is seen invading into the anterior chest wall on the right and involving the pectoralis major muscle. The mass is largely homogeneous in enhancement and conforms to the shape of the vessels. Chamberlain procedure was performed and tissue biopsy showed composite lymphoma consisting of nodular sclerosing Hodgkin lymphoma and peripheral T-cell lymphoma.

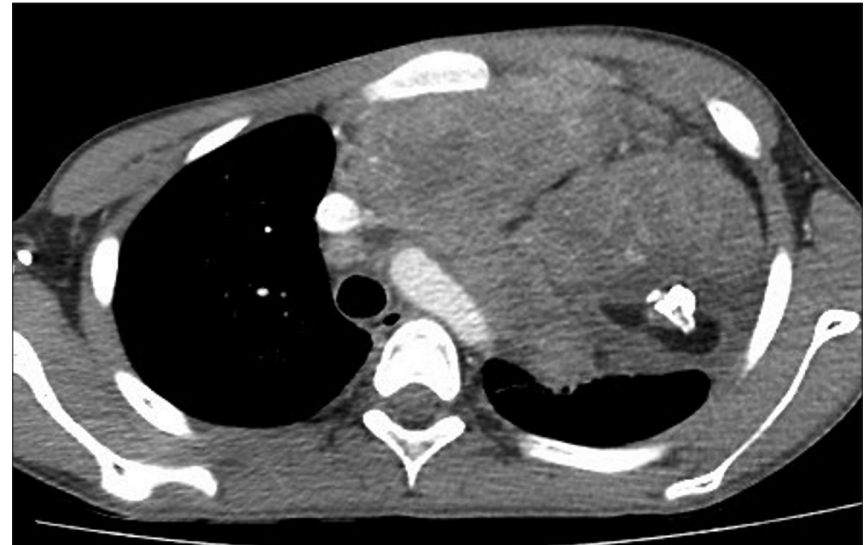

Fig. 5 Axial contrast-enhanced CT image shows a large mediastinal mass compressing the left lung of a 16-year-old boy who presented with cough of one month's duration and breathlessness, probably causing his symptoms. Fat content and a tooth are seen within the predominantly solid mass, consistent with a mature teratoma. The diagnosis was confirmed histologically.
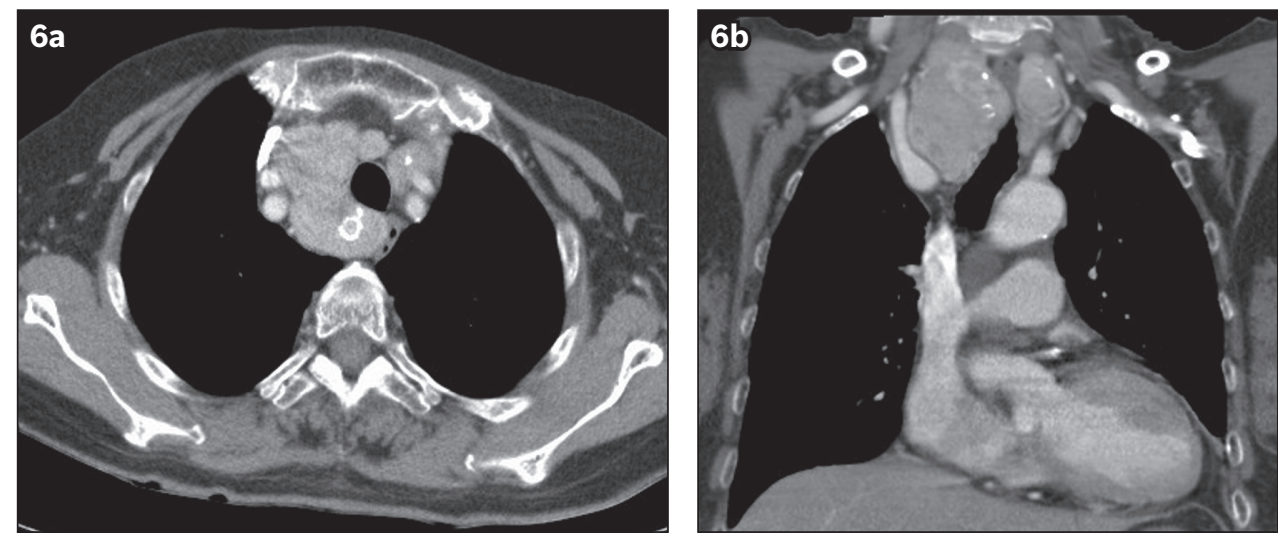

Fig. 6 A neck mass that was gradually increasing in size in a 71-year-old woman. (a) Axial and (b) coronal contrast-enhanced CT images show nodular heterogenous thyroid goitre with retrosternal extension into the upper mediastinum. Fine needle aspiration cytology favoured nodular goitre with cystic changes.

Primary mediastinal lymphoma (Fig. 4) also occurs commonly in the anterior mediastinum. It shows mild-to-moderate contrast enhancement, with irregular contours and surface lobulation. It can infiltrate the surrounding structures, such as the vessels, resulting in encasement. Haemorrhage, necrosis and cystic degeneration can also be present. Lymphoma often presents with pleural or pericardial effusion and other enlarged lymph nodes. ${ }^{(11}$

On CT, teratomas are distinct in appearance, with fluid, soft tissue, calcification and fat content (Fig. 5), and well demarcated with lobulated outlines. Tissue organisation varies; the calcification may be linear, punctate, flocculent, or appear as teeth or bone. Occasionally, mature teratomas can appear as purely cystic lesions, making diagnosis more challenging. The presence of a fat-fluid level is a highly specific sign. ${ }^{(12)}$

A retrosternal thyroid goitre extension may be mistaken for a mediastinal mass on chest radiography, but should be fairly obvious on $\mathrm{CT}$, as it is contiguous with the thyroid gland (Fig. 6). On plain radiographs, the cervicothoracic sign is useful to differentiate masses originating in the anterior mediastinum from those arising from the posterior mediastinum or neck. This sign occurs when the mass extends superior to the clavicles. As the anterior mediastinum ends at the level of the clavicles, any mass seen above the clavicles is either in the posterior mediastinum or in the neck.

In conclusion, SVC aneurysm is a rare cause of an anterior mediastinal mass that can be mistaken for one of the more common mediastinal masses. However, careful evaluation with contrast-enhanced CT can usually reveal the vascular nature of the lesion. CT angiography, with a combination of arterial and venous phases, can help to further confirm this. This is essential to avoid biopsy of the lesion, which would be catastrophic, and is also useful for planning of management.

\section{ACKNOWLEDGEMENT}

The authors would like to thank Dr Sandeep Halagatti Venkatesh for his contribution to the case. 
ABSTRACT A 68-year-old woman presented with weight loss of $7 \mathrm{~kg}$ over two years. Clinical examination revealed no specific abnormality and the patient was otherwise asymptomatic. Chest radiography performed to screen for malignancy revealed a soft tissue opacity projected over the mediastinum. Computed tomography of the thorax showed an enhancing anterior mediastinal mass with heterogeneous enhancement and contrast pooling in the arterial phase, followed by homogeneous enhancement in the venous phase, consistent with an aneurysm arising from the superior vena cava. This case highlights superior vena cava aneurysms as extremely rare causes of anterior mediastinal masses. Other causes of anterior mediastinal masses were also discussed.

Keywords: aneurysm, mediastinal mass, superior vena cava

\section{REFERENCES}

1. Yokoyama T, Setoguchi T, Sakamoto K, et al. A saccular superior vena cava aneurysm with unique diagnostic computed tomography findings. Respirol Case Rep 2017; 6:e00281.
2. Taira A, Akita H. Ruptured venous aneurysm of the persistent left superior vena cava. Angiology 1981; 32:656-9.

3. Oh SG, Kim KH, Seon HJ, et al. Unusual cause of acute right ventricular dysfunction: rapid progression of superior vena cava aneurysm complicated by thrombosis and pulmonary thromboembolism. J Korean Med Sci 2011; 26:690-3.

4. Janczak D, Skiba J, Gemel M, et al. Giant saccular superior vena cava aneurysm-a rare and difficult clinical case. J Thorac Dis 2016; 8:E247-9.

5. Patel A, Cobb R, Rivera V, Simpson S. Untreated superior vena cava aneurysm: radiological significance and review of the literature. Case Rep Radiol 2016; 2016:6960757.

6. Varma PK, Dharan BS, Ramachandran P, Neelakandhan KS. Superior vena caval aneurysm. Interact Cardiovasc Thorac Surg 2003; 2:331-3.

7. Griviau L, Chevallier O, Favelier S, et al. Endovascular management of a large aneurysm of the superior vena cava involving internal thoracic vein with remodeling technique. Quant Imaging Med Surg 2016; 6:315-7.

8. Jargiello T, Durakiewicz M, Sojka M, Czekajska-Chehab E, SzczerboTrojanowska M. Saccular aneurysm of superior vena cava treated with percutaneous, transcatheter thrombin injection. Cardiovasc Intervent Radiol $2014 ; 37: 529-32$

9. Hidvegi RS, Modry DL, LaFléche L. Congenital saccular aneurysm of the superior vena cava: radiolographic features. AJR Am J Roentgenol 1979; 133:924-7.

10. Benveniste MF, Rosado-de-Christenson ML, Sabloff BS, et al. Role of imaging in the diagnosis, staging, and treatment of thymoma. Radiographics 2011; 31:1847-63.

11. Shaffer K, Smith D, Kirn D, et al. Primary mediastinal large-B-cell lymphoma: radiologic findings at presentation. AJR Am J Roentgenol 1996; 167:425-30.

12. Moeller KH, Rosado-de-Christenson ML, Templeton PA. Mediastinal mature teratoma: imaging features. AJR Am J Roentgenol 1997; 169:985-90. 


\section{SINGAPORE MEDICAL COUNCIL CATEGORY 3B CME PROGRAMME} (Code SMJ 201901B)

Question 1. What are the complications of superior vena cava (SVC) aneurysms?
(a) Rupture.
(b) Dissection.
(c) Intramural haematoma.
(d) Pulmonary embolism.

Question 2. For which lesions would the hilum overlay sign be evident?
(a) Perihilar lung mass.
(b) Paravertebral mass.
(c) Hilar enlarged lymph node.
(d) Thymoma.

Question 3. What is the typical computed tomography enhancement pattern of an SVC aneurysm?
(a) Heterogeneous in the venous phase.
(b) Enhances homogeneously in the arterial phase following the aorta.
(c) Enhances homogenously in the venous phase following the SVC.
(d) Shows contrast pooling in the dependent portion of the aneurysm in the arterial phase.

Question 4. Which is a differential diagnosis for anterior mediastinal mass?
(a) Lymphoma.
(b) Aortic aneurysm.
(c) Thyroid goitre.
(d) Oesophageal squamous cell carcinoma.

Question 5. For which cases of SVC aneurysm are surgical interventions advocated?
(a) Aneurysms more than $40 \mathrm{~mm}$ in diameter.
(b) Fusiform aneurysms.
(c) Asymptomatic aneurysms.
(d) Thrombosed aneurysms.

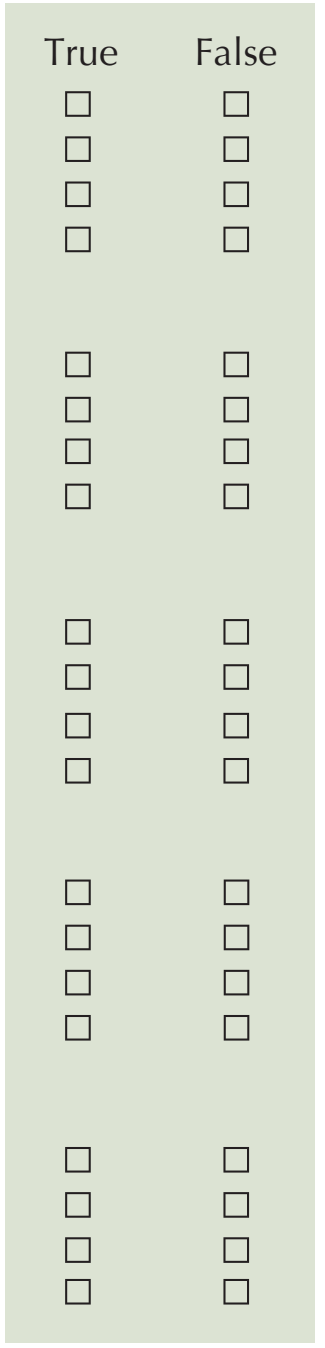

\section{Doctor's particulars:}

Name in full:

Specialty:
MCR no.:

Email:

\footnotetext{
SUBMISSION INSTRUCTIONS:

Visit the SMJ website: http://www.smj.org.sg/current-issue and select the appropriate quiz. You will be redirected to the SMA login page.

For SMA member: (1) Log in with your username and password (if you do not know your password, please click on 'Forgot your password?'). (2) Select your answers for each quiz and click 'Submit'.

For non-SMA member: (1) Create an SMJ CME account, or log in with your SMJ CME username and password (for returning users). (2) Make payment of SGD 21.40 (inclusive of $7 \%$ GST) via PayPal to access this month's quizzes. (3) Select your answers for each quiz and click 'Submit'.

RESULTS:

(1) Answers will be published online in the SMJ March 2019 issue. (2) The MCR numbers of successful candidates will be posted online at the SMJ website by 8 March 2019 (3) Passing mark is $60 \%$. No mark will be deducted for incorrect answers. (4) The SMJ editorial office will submit the list of successful candidates to the Singapore Medical Council. (5) One CME point is awarded for successful candidates. (6) SMC credits CME points according to the month of publication of the CME article (i.e. points awarded for a quiz published in the December 2017 issue will be credited for the month of December 2017, even if the deadline is in January 2018).

Deadline for submission (January 2019 SMJ 3B CME programme): 12 noon, 1 March 2019.
} 